\title{
New poly( $p$-phenylenevinylene) derivatives containing isosorbide unit in the side-chain
}

\author{
H. Zrida ${ }^{1}$, K. Hriz ${ }^{1}$, N. Jaballah ${ }^{1}$, N. Sakly ${ }^{1}$, D. Kreher ${ }^{2}$, M. Majdoub ${ }^{*}$ \\ ${ }^{1}$ Laboratoire des Interfaces et des Matériaux Avancés (LIMA), Faculté des Sciences de Monastir, Bd. \\ de l'Environnement, 5019 Monastir, Université de Monastir, Tunisia \\ ${ }^{2}$ Laboratoire de Chimie des polymères Université Pierre et Marie-Currie (UPMC), 4 Place Jussieu, 75005 Paris, France
}

Received 10 March 2014; accepted in revised form 18 May 2014

\begin{abstract}
New conjugated PPV derivatives containing the chiral isosorbide group (P1-3) have been synthesized via the Gilch reaction. The polymers are optically active, soluble in common organic solvents and show good film-forming abilities. High number-average molecular weights were determined by size exclusion chromatography (SEC) $\left(16 \cdot 10^{3}-\right.$ $\left.21 \cdot 10^{3} \mathrm{~g} \cdot \mathrm{mol}^{-1}\right)$. The molecular structures of the polymers were confirmed by nuclear magnetic resonance (NMR) and Fourier transform infrared (FTIR) spectroscopies. Thermogravimetric analysis of the polymers showed good thermal stability up to $320^{\circ} \mathrm{C}$. The optical properties of these $\pi$-conjugated materials were investigated by UV-vis absorption and photoluminescence (PL) spectroscopies. The polymers show a yellow fluorescence in dilute solution, and an orange emission is observed in thin films. The introduction of the polar isosorbide groups improved the PL intensity, and quantum yields between 50 and $73 \%$ were obtained. The HOMO-LUMO energy levels were estimated by cyclic voltammetry, and the electrochemical gaps were 1.81, 1.83 and $2.48 \mathrm{eV}$ for P1, P2 and P3, respectively. Single-layer diode devices were fabricated and show relatively low turn-on voltages between 3.1 and $3.4 \mathrm{~V}$.
\end{abstract}

Keywords: polymer synthesis, optically active polymers, semi-conducting polymers, isosorbide, photoluminescence

\section{Introduction}

The discovery of electroluminescence in poly ( $p$ phenylenevinylene) (PPV) in 1990 [1] created a new domain in polymeric material applications. Since then, enormous progress has been made in the macromolecular engineering of the $\pi$-conjugated polymers and in their uses as active materials in polymeric light-emitting diodes (PLEDs) [2-6]. These polymers are promising organic analogues of inorganic semi-conducting materials, and their exploitation in other electronic devices, such as thin-film transistors [7, 8], photovoltaic cells $[9,10]$, chemical sensors [11] and organic lasers [12] are currently expanding. The main advantages of using such functional polymers lie in their low production cost and easy processibility. In fact, these materials are com- patible with solution processing techniques, thus eliminating the vacuum deposition steps usually required for the elaboration of inorganic semi-conducting thin layers. Solution processing also expands the repertoire of tolerant substrates and processing options, allowing flexible plastics to be used in combination with relatively simple methods such as spin coating and inkjet printing. Though, the major characteristic of the semi-conducting polymers is their adjustable macormolecular structure and consequently there tuneable opto-electronic properties [13-16].

Among $\pi$-conjugated polymers, the poly ( $p$ phenylenevinylene) (PPV) and its derivatives are the most studied and exploited $[17,18]$. Due to the high rigidity of the macromolecular structure, PPV is

\footnotetext{
*Corresponding author, e-mail: mustapha.majdoub@fsm.rnu.tn (C) BME-PT
} 
insoluble in common organic solvents. This intractability has been addressed by chemically attaching aliphatic side-chains to the polymer backbone [19]. Hence, many PPV-type architectures are processed in a derivative form. The effects of the side-group structure on the opto-electronic properties have been extensively investigated [20]. However, most studies have involved nonpolar sidechains, and the effect of relatively polar side-groups was rarely reported (e.g PPV derivative containing ethylene oxide-type side chains) [12]. Here, we report the first PPV derivatives containing the polar and chiral isosorbide groups. In fact, the incorporation of chiral groups in polypyrroles and polythiophenes was reported. These groups confer original physico-chemical properties to the polymer [2123]. Therefore, these optically active polymers were used to prepare chiral electrodes for asymmetric electrosynthesis, polarization-sensitive electro-optical devices, polarized photo- and electroluminescent devices and enantioselective sensors [24]. Herein, we present the synthesis and structural characterizations of the isosorbide-containing PPVs; the thermal, thin film surface, optical and electrochemical properties were investigated.

\section{Experimental}

\subsection{Materials and measurements}

The poly (2-hexyloxy-5-methoxy-p-phenylenevinylene) (MH-PPV) was synthesized by using Gilch condensation. Detailed synthesis procedure can be found elsewhere [20]. Isosorbide (98\%, Acros Organics, France), 4-toluenesulfonyl chloride (98\%, SigmaAldrich, France), 4-methoxyphenol (99\%, Acros Organics, France ), ethyl bromide (98\%, Acros Organics, France), hexyl bromide (98\%, Acros Organics, France), dodecyl bromide (98\%, Acros Organics, France), potassium carbonate (99\%, Acros Organics, France), potassium tert-butoxide (98\%, Acros Organics, France), $t$-butanol (Acros Organics, France), sodium hydride (60\% dispersion in mineral oil, Sigma-Aldrich, France), paraformaldehyde (96\%, Acros Organics, France), sodium (Sigma Aldrich, France) and dimethylformamide (DMF) (Acros Organics, France) were used as received. Ethanol was dried over $\mathrm{Mg} / \mathrm{I}_{2}$ and freshly distilled before use. All the solvents used in this study were purchased from Sigma-Aldrich (France). Optical rotations were measured with Atago Polax-2L digital polarimeter (Atago, Japan). ${ }^{1} \mathrm{H}-\mathrm{NMR}$ and ${ }^{13} \mathrm{C}$ -
NMR spectral data were obtained on a Bruker AV 300 spectrometer (Biospin, Strasbourg, France). Fourier transform infrared (FTIR) spectra were acquired on a Perkin-Elmer BX FTIR (Perkin-Elmer, California, USA) system spectrometer by dispersing samples in $\mathrm{KBr}$ disks. Size exclusion chromatography (SEC) was performed on an Agilent Technologies 1200 HPLC (Agilent, France). The experiment was done at room temperature using THF as eluent with standard polystyrene calibration. Thermogravimetric analysis (TGA) was carried out on TA Instruments Q50 (TA Instruments, USA) under nitrogen at a heating rate of $10^{\circ} \mathrm{C} \cdot \mathrm{min}^{-1}$. DSC was performed on a Mettler Toledo DSC1(Mettler Toledo, France) with a heating rate of $10^{\circ} \mathrm{C} \cdot \mathrm{min}^{-1}$. UV-vis absorption spectra were recorded on a Cary 300 spectrophotometer (Agilent, France). Fluorescence spectra were obtained on a Jobin-Yvon spectrometer HR460 (HORIB, Kyoto, Japan) coupled to a nitrogencooled Si charged-coupled device (CCD). Samples were excited at $365 \mathrm{~nm}$ with a $450 \mathrm{~W}$ xenon lamp.

The spectral sensitivity of the measurement system was calibrated using tungsten standard lamp. The PL quantum yields were measured in dilute chloroform solution according to a relative method using quinine sulfate (Sigma-Aldrich, France) $\left(10^{-5} \mathrm{M}\right.$ solution of $0.5 \mathrm{H}_{2} \mathrm{SO}_{4}$ ) [25]. Absorbance of the sample solutions was kept below 0.05 to avoid inner filter effect, and measurements were performed at room temperature using freshly prepared solutions. Both sample and reference solutions were excited at the same wavelength $(365 \mathrm{~nm})$, and the PL quantum efficiency of the quinine sulfate solution $\left(\Phi_{\mathrm{r}}\right)$ was assumed to be 0.54 [26]. Hence, the PL quantum efficiency of the sample $\left(\Phi_{\mathrm{s}}\right)$ can be calculated using Equation (1):

$\frac{\Phi_{\mathrm{s}}}{\Phi_{\mathrm{r}}}=\frac{A_{\mathrm{r}}}{A_{\mathrm{s}}} \frac{F_{\mathrm{s}}}{F_{\mathrm{r}}} \frac{n_{\mathrm{s}}^{2}}{n_{\mathrm{r}}^{2}}$

where $A_{\mathrm{r}}$ and $F_{\mathrm{r}}$ are the absorbance at the excitation wavelength and emission integration area for the reference, $A_{\mathrm{S}}$ and $F_{\mathrm{S}}$ are the absorbance and emission integral for the sample, while $n_{\mathrm{s}}$ and $n_{\mathrm{r}}$ are the refractive index of the polymer solution and quinine sulfate solution, respectively. For solid state optical measurements, the film was spin-coated onto a quartz substrate from $50 \mu \mathrm{L}$ of a chloroform solution $\left(2 \cdot 10^{-2} \mathrm{M}\right)$. The film thicknesses were measured by a Dektak profilometer (Sloan, USA) and were about $60 \mathrm{~nm}$. Film thickness was controlled 
by spinning rate to obtain comparable thicknesses and so similar refractive indices. Thus, the ratio of PL quantum yields of three film samples can be calculated according to Equation (2):

$\frac{\Phi_{\mathrm{s}}}{\Phi_{\mathrm{r}}}=\frac{A_{\mathrm{r}}}{A_{\mathrm{s}}} \frac{F_{\mathrm{s}}}{F_{\mathrm{r}}}$

The atomic force microscopy (AFM) analyses of the polymer coated glass surfaces were carried out using a Nanoscope III (Digital Instruments, Santa Barbara, CA) operating in the tapping mode. Contact angle measurements with three different liquids (DI water, formamide and diiodomethane) were performed with Digidrop (GBX, France) contact angle instrument. Every reported contact angle measurement represents an average value of at least three separated drops on different areas of the given wafer. The size and volume of the drops were kept constant. Cyclic voltammetry (CV) was performed on a $\mathrm{CHI}$ 660B electrochemical station (CH Instruments Inc., USA) in a three-electrode cell and using material films that were drop-cast onto an indium tin oxide (ITO/1 $\mathrm{cm}^{2}$ ) working electrode. The measurements were carried out at a scanning rate of $50 \mathrm{mV} \cdot \mathrm{s}^{-1}$ against an $\mathrm{Ag} / \mathrm{AgCl}$ reference electrode, a counter electrode made with a Platinum wire ( $1 \mathrm{~cm}$ of length) using $0.1 \mathrm{M}$ tetrabutylammoniumfluoroborate $((n-$ $\mathrm{Bu})_{4} \mathrm{NBF}_{4}$ ) in acetonitrile as supporting electrolyte. The electrochemical cell was externally calibrated by ferrocene under the same conditions as the polymers. The measurements were performed at $25^{\circ} \mathrm{C}$, and the cell was deoxygenated with argon before each reductive scan.

\subsection{Synthesis of the monomers (e1-3)}

Synthesis of 2,5-O-ditosyl-1,4:3,6-dianhydro-Dsorbitol (a)

$120 \mathrm{~mL}$ of $5 \mathrm{M}$ aqueous $\mathrm{NaOH}$ was added to $100 \mathrm{~mL}$ of $2 \mathrm{M}$ isosorbide/THF solution. The mixture was cooled to $5^{\circ} \mathrm{C}$. A solution of 4-toluenesulfonyl chloride $(0.38 \mathrm{~mol})$ in $100 \mathrm{~mL}$ of THF was added dropwise. After $2 \mathrm{~h}$, the mixture was placed in a separating funnel and the phases were separated. The aqueous phase was extracted with dichloromethane $(3 \times 50 \mathrm{~mL})$. The organic phases were combined and washed with water, dried over $\mathrm{MgSO}_{4}$ and the solvent was evaporated. The product was obtained as a white solid. M.p: $98^{\circ} \mathrm{C}$; yield: $95 \%$; ${ }^{1} \mathrm{H}-\mathrm{NMR}$ (300 MHz, $\left.\mathrm{CDCl}_{3}, \delta\right): 7.80-7.74$ (m, 4H, Ar-H),
7.36-7.27 (m, 4H, Ar-H), 4.87-4.83 (m, 2H, $\left.\mathrm{H}_{2}, \mathrm{H}_{5}\right)$, $4.61\left(\mathrm{~m}, 1 \mathrm{H}, \mathrm{H}_{3}\right), 4.46\left(\mathrm{~d}, \mathrm{~J}=3 \mathrm{~Hz}, 1 \mathrm{H}, \mathrm{H}_{4}\right), 3.96-$ $3.80\left(\mathrm{~m}, 3 \mathrm{H}, \mathrm{H}_{1}{ }^{\mathrm{b}}, \mathrm{H}_{6}{ }^{\mathrm{a}}, \mathrm{H}_{6}{ }^{\mathrm{b}}\right), 3.65\left(\mathrm{~m}, 1 \mathrm{H}, \mathrm{H}_{1}{ }^{\mathrm{a}}\right), 2.46$ (s, $\left.6 \mathrm{H}, \mathrm{SO}_{2} \mathrm{C}_{6} \mathrm{H}_{4} \mathrm{CH}_{3}\right) ;{ }^{13} \mathrm{C}-\mathrm{NMR}\left(75.5 \mathrm{MHz}, \mathrm{CDCl}_{3}\right.$,

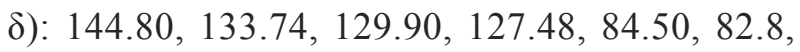
$83.70,80.5,73.30,71.20,21.62$.

\section{Synthesis of 1,4:3,6-dianhydro-5-[4-} methoxyphenyloxy]-2-O-tosyl-5-deoxy-L-iditol (b) A mixture of 4-methoxyphenol (10 mmol), potassium carbonate $(10 \mathrm{mmol})$ and 2,5-O-ditosyl-1,4:3,6dianhydro-D-sorbitol (a) (10 mmol) was stirred in $20 \mathrm{~mL}$ of DMF at $60^{\circ} \mathrm{C}$. After $24 \mathrm{~h}$ the reaction mixture was poured into distilled water and extracted with dichloromethane. The extract was washed with distilled water, dried over anhydrous $\mathrm{MgSO}_{4}$ and concentrated at reduced pressure. The resultant crude product was purified by recrystallization from chloroform/ethanol $(1: 5 \mathrm{v} / \mathrm{v})$. The product is obtained as a white powder. M.p: $91^{\circ} \mathrm{C}$; yield: $60 \% ;{ }^{1} \mathrm{H}-\mathrm{NMR}$ $\left(300 \mathrm{MHz}, \mathrm{CDCl}_{3}, \delta\right): 7.82(\mathrm{~d}, \mathrm{~J}=9.0 \mathrm{~Hz}, 2 \mathrm{H}, \mathrm{Ar}-\mathrm{H})$, 7.39 (d, J = 9.0 Hz, 2H, Ar-H), 6.83 (s, 4H, Ar-H), $4.94\left(\mathrm{~d}, \mathrm{~J}=3 \mathrm{~Hz}, 1 \mathrm{H}, \mathrm{H}_{2}\right), 4.74-4.67\left(\mathrm{~m}, 3 \mathrm{H}, \mathrm{H}_{3}, \mathrm{H}_{4}\right.$, $\left.\mathrm{H}_{5}\right), 4.04-3.88\left(\mathrm{~m}, 4 \mathrm{H}, \mathrm{H}_{1}{ }^{\mathrm{b}}, \mathrm{H}_{1}{ }^{\mathrm{a}}, \mathrm{H}_{6}{ }^{\mathrm{b}}, \mathrm{H}_{6}{ }^{\mathrm{a}}\right), 3.77$ (s, $\left.3 \mathrm{H}, \mathrm{OCH}_{3}\right), 2.47\left(\mathrm{~s}, 3 \mathrm{H}, \mathrm{CH}_{3}\right) ;{ }^{13} \mathrm{C}-\mathrm{NMR}(75 \mathrm{MHz}$, $\left.\mathrm{CDCl}_{3}, \delta\right): 152.59,148.78,143.53,128.25,126.01$, $114.60,112.93,83.49,83.41,81.09,79.52,70.67$, $70.28,53.83,19.86$.

\section{Synthesis of 1,4:3,6-dianhydro-5-[4-}

methoxyphenyloxy]-5-deoxy-L-iditol (c)

The 1,4:3,6-dianhydro-5-[4-methoxyphenyloxy]-2O-tosyl-5-deoxy-L-iditol (b) $(10 \mathrm{mmol})$ was refluxed with sodium ethoxide in ethanol $(1 \mathrm{M})$ for $4 \mathrm{~h}$. After cooling to room temperature, the solution was concentrated. The residue was then poured into distilled water and extracted with dichloromethane. The organic layer was washed several times with distilled water and dried over anhydrous $\mathrm{MgSO}_{4}$. The product was recuperated as a white powder by precipitation in petroleum ether. M.p: $82^{\circ} \mathrm{C}$; yield $85 \%$; ${ }^{1} \mathrm{H}-\mathrm{NMR}\left(300 \mathrm{MHz}, \mathrm{CDCl}_{3}, \delta\right): 6.90-6.82(\mathrm{~m}, 4 \mathrm{H}$, $\operatorname{Ar}-\mathrm{H}), 4.78\left(\mathrm{~d}, \mathrm{~J}=3.9 \mathrm{~Hz}, 1 \mathrm{H}, \mathrm{H}_{5}\right), 4.71\left(\mathrm{~m}, 1 \mathrm{H}, \mathrm{H}_{4}\right)$, $4.62\left(\mathrm{~d}, \mathrm{~J}=3.6 \mathrm{~Hz}, 1 \mathrm{H}, \mathrm{H}_{3}\right), 4.36\left(\mathrm{~m}, 1 \mathrm{H}, \mathrm{H}_{2}\right), 4.05-$ $3.89\left(\mathrm{~m}, 4 \mathrm{H}, \mathrm{H}_{1}{ }^{\mathrm{a}}, \mathrm{H}_{1}{ }^{\mathrm{b}}, \mathrm{H}_{6}{ }^{\mathrm{a}}, \mathrm{H}_{6}{ }^{\mathrm{b}}\right), 3.77\left(\mathrm{~s}, 3 \mathrm{H}, \mathrm{OCH}_{3}\right)$, 2.5 (s, $1 \mathrm{H}, \mathrm{OH}) ;{ }^{13} \mathrm{C}$-NMR $\left(75 \mathrm{MHz}, \mathrm{CDCl}_{3}, \delta\right.$ ): 154.33, 150.92, 116.48, 114.80, 87.88, 85.12, 81.73, 75.92, 74.49, 72.29, 55.71; FTIR $\left(\mathrm{cm}^{-1}\right): 3400(\mathrm{w}$, $\mathrm{C}-\mathrm{H}$, stretching). 
Synthesis of the 1,4:3,6-dianhydro-2-O-alkyl-5-[4methoxyphenyloxy]-5-deoxy-L-iditols (d1-3)

A solution of 1,4:3,6-dianhydro-5-[4-methoxyphenyloxy]-5-deoxy-L-iditol (c) $(10 \mathrm{mmol})$ in $10 \mathrm{~mL}$ of dry DMF was added dropwise under argon to a suspension of $\mathrm{NaH}(15 \mathrm{mmol})$ of a $60 \%$ dispersion in mineral oil; washed with hexane) in $10 \mathrm{~mL}$ of dry DMF. After stirring for 30 minutes at $0^{\circ} \mathrm{C}$, a $12 \mathrm{mmol}$ of alkyl bromide was added. The reaction mixture was stirred for $24 \mathrm{~h}$ at room temperature. The resulting mixture was then poured into distilled water and extracted with dichloromethane. The organic layer was washed several times with distilled water, then dried over anhydrous $\mathrm{MgSO}_{4}$ and evaporated. The resultant crude product was purified by column chromatography with cyclohexane/ethyl acetate (90:10: v/v).

d1: $\mathrm{R}=$ ethyl; aspect: colorless oil; yield: $89 \%$; ${ }^{1} \mathrm{H}-\mathrm{NMR}$ (300 MHz, DMSO, $\delta$ ): 6.90-6.88 (m, 4H, Ar-H), $4.73\left(\mathrm{~m}, 1 \mathrm{H}, \mathrm{H}_{5}\right), 4.58\left(\mathrm{~d}, \mathrm{~J}=6 \mathrm{~Hz}, 1 \mathrm{H}, \mathrm{H}_{4}\right)$, $4.51\left(\mathrm{~d}, \mathrm{~J}=6 \mathrm{~Hz}, 1 \mathrm{H}, \mathrm{H}_{3}\right), 3.95-3.78\left(\mathrm{~m}, 5 \mathrm{H}, \mathrm{H}_{2}, \mathrm{H}_{1}^{\mathrm{a}}\right.$, $\left.\mathrm{H}_{1}{ }^{\mathrm{b}}, \mathrm{H}_{6}{ }^{\mathrm{a}}, \mathrm{H}_{6}{ }^{\mathrm{b}}\right), 3.70\left(\mathrm{~s}, 3 \mathrm{H}, \mathrm{OCH}_{3}\right), 3.53(\mathrm{~m}, 2 \mathrm{H}$, $\left.-\mathrm{OCH}_{2}\right), 1.12\left(\mathrm{t}, \mathrm{J}=6 \mathrm{~Hz}, 3 \mathrm{H}, \mathrm{CH}_{3}\right) ;{ }^{13} \mathrm{C}-\mathrm{NMR}$ (75 MHz, DMSO, $\delta$ ): 153.83, 150.54, 116.47, 114.71, $85.18,84.86,82.63,81.39,71.57,71.28,64.06$, $55.29,15.11$.

d2: $\mathrm{R}=$ hexyl; aspect colorless oil; yield: $85 \%$. ${ }^{1} \mathrm{H}-\mathrm{NMR}\left(300 \mathrm{MHz}, \mathrm{CDCl}_{3}, \delta\right)$ : 6.91-6.83 (m, 4H, Ar-H), 4.72-4.67 (m, 3H, H3, $\left.\mathrm{H}_{4}, \mathrm{H}_{5}\right), 4.03-3.90$ (m, $\left.5 \mathrm{H}, \mathrm{H}_{1}{ }^{\mathrm{a}}, \mathrm{H}_{1}{ }^{\mathrm{b}}, \mathrm{H}_{6}{ }^{\mathrm{a}}, \mathrm{H}_{6}{ }^{\mathrm{b}}, \mathrm{H}_{2}\right), 3.78\left(\mathrm{~s}, 3 \mathrm{H}, \mathrm{OCH}_{3}\right)$, $3.52\left(\mathrm{td},{ }^{4} \mathrm{~J}=3 \mathrm{~Hz},{ }^{3} \mathrm{~J}=9 \mathrm{~Hz}, 2 \mathrm{H},-\mathrm{OCH}_{2}\right), 1.63(\mathrm{~m}$, $\left.2 \mathrm{H}, \quad \mathrm{OCH}_{2}-\mathrm{CH}_{2}-\mathrm{CH}_{2}\right), \quad 1.36-1.30(\mathrm{~m}, \quad 6 \mathrm{H}$, $\left.-\left(\mathrm{CH}_{2}\right)_{3}-\mathrm{CH}_{3}\right), 0.92\left(\mathrm{t}, \mathrm{J}=7.8 \mathrm{~Hz}, 3 \mathrm{H},-\mathrm{CH}_{3}\right) ;{ }^{13} \mathrm{C}-$ NMR (75 MHz, $\left.\mathrm{CDCl}_{3}, \delta\right): 154.56,151.29,116.64$, $115,85.98,85.56,83.68,82.06,72.62,72.39$, 70.12, 55.89, 31.80, 29.90, 25.93, 22.76, 14.19.

d3: $\mathrm{R}=$ dodecyl; aspect: white solid. M.p.: $41^{\circ} \mathrm{C}$; yield: $75 \%$; ${ }^{1} \mathrm{H}-\mathrm{NMR}\left(300 \mathrm{MHz}, \mathrm{CDCl}_{3}, \delta\right): 6.91-$ $6.83(\mathrm{~m}, 4 \mathrm{H}, \mathrm{Ar}-\mathrm{H}), 4.72-4.67\left(\mathrm{~m}, 3 \mathrm{H}, \mathrm{H}_{3}, \mathrm{H}_{4}, \mathrm{H}_{5}\right)$, 4.03-3.91 (m, 5H, $\left.\mathrm{H}_{1}{ }^{\mathrm{a}}, \mathrm{H}_{1}{ }^{\mathrm{b}}, \mathrm{H}_{6}{ }^{\mathrm{a}}, \mathrm{H}_{6}{ }^{\mathrm{b}}, \mathrm{H}_{2}\right), 3.78$ (s, $\left.3 \mathrm{H}, \mathrm{OCH}_{3}\right), 3.54\left(\mathrm{td},{ }^{4} \mathrm{~J}=5.4 \mathrm{~Hz},{ }^{3} \mathrm{~J}=9 \mathrm{~Hz}, 2 \mathrm{H}\right.$, $\left.\mathrm{OCH}_{2}\right), 1.57\left(\mathrm{~m}, 2 \mathrm{H}, \mathrm{OCH}_{2}-\mathrm{CH}_{2}\right), 1.27(\mathrm{~m}, 18 \mathrm{H}$, $\left.-\left(\mathrm{CH}_{2}\right)_{9}-\mathrm{CH}_{3}\right), 0.92\left(\mathrm{t}, \mathrm{J}=6 \mathrm{~Hz}, 3 \mathrm{H}, \mathrm{CH}_{3}\right) ;{ }^{13} \mathrm{C}-\mathrm{NMR}$ (75 MHz, $\mathrm{CDCl}_{3}, \delta$ ): 153.85, 150.58, 115.94, 114.31, $85.27,84.85,82.98,81.35,71.94,71.70,69.45$, 55.20, 31.40, 29.24, 29.13, 29.11, 29.08, 29.05, $28.91,28.83,25.57,22.17,13.59$.
Synthesis of the dichloromethyl aromatic derivatives (e1-3)

A mixture of the corresponding 1,4:3,6-dianhydro2-O-alkyl-5-[4-methoxyphenyloxy]-5-deoxy-L-iditols (d1-3) (10 mmol), paraformaldehyde (15 mmol) and $37 \%$ aqueous $\mathrm{HCl}(16 \mathrm{mmol})$ in acetic acid was stirred at $60^{\circ} \mathrm{C}$. The progress of the reaction was monitored by TLC ( $\mathrm{R}$ = ethyl, hexyl or dodecyl; eluent: cyclohexane/acetate $6 / 1 \mathrm{v} / \mathrm{v}$ ). The resulting mixture was then poured into distilled water and extracted with dichloromethane. The organic layer was washed several times with distilled water, then dried over anhydrous $\mathrm{MgSO}_{4}$ and concentrated at reduced pressure. The resultant crude product was purified by recrystallization from an appropriate solvent.

e1: $\mathrm{R}=$ ethyl; recrystallization from dichloromethane/ethanol $(1: 5 \mathrm{v} / \mathrm{v})$; aspect: white solid; yield: $85 \%$; m.p.: $98^{\circ} \mathrm{C} ;[\alpha]_{\mathrm{D}}=42.5^{\circ}\left(2, \mathrm{CHCl}_{3}\right) ;{ }^{1} \mathrm{H}-\mathrm{NMR}$ $\left(300 \mathrm{MHz}, \mathrm{CDCl}_{3}, \delta\right): 7.01$ (s, 1H, Ar-H), 6.94 (s, $1 \mathrm{H}, \mathrm{Ar}-\mathrm{H}), 4.82\left(\mathrm{~m}, 1 \mathrm{H}, \mathrm{H}_{5}\right), 4.76-4.72\left(\mathrm{~m}, 2 \mathrm{H}, \mathrm{H}_{3}\right.$, $\left.\mathrm{H}_{4}\right), 4.67-4.57$ (m, 4H, $\left.\mathrm{CH}_{2}-\mathrm{Cl}\right), 4.05-3.88(\mathrm{~m}, 8 \mathrm{H}$, $\left.\mathrm{H}_{1}{ }^{\mathrm{a}}, \mathrm{H}_{1}{ }^{\mathrm{b}}, \mathrm{H}_{6}{ }^{\mathrm{a}}, \mathrm{H}_{6}{ }^{\mathrm{b}}, \mathrm{H}_{2}, \mathrm{OCH}_{3}\right), 3.53\left(\mathrm{~m}, 2 \mathrm{H}, \mathrm{OCH}_{2}\right)$, $1.25\left(\mathrm{t}, \mathrm{J}=6.9 \mathrm{~Hz}, 3 \mathrm{H}, \mathrm{CH}_{3}\right) ;{ }^{13} \mathrm{C}-\mathrm{NMR}(75 \mathrm{MHz}$, $\left.\mathrm{CDCl}_{3}, \delta\right): 151.82,148.37,127.96,127.17,115.55$, $113.45,85.85,85.25,83.26,82.23,72.51,72.06$, 65.18, 56.21, 41.06, 41.02, 15.28; FTIR $\left(\mathrm{cm}^{-1}\right): 670$ (s, $\mathrm{C}-\mathrm{Cl}$ stretching).

e2: $\mathrm{R}=$ hexyl; recrystallization from dichloromethane/ethanol (1:5 v/v); aspect: white solid; yield: $80 \%$; m.p.: $70^{\circ} \mathrm{C}$; $[\alpha]_{\mathrm{D}}=47.5^{\circ}\left(2, \mathrm{CHCl}_{3}\right) ;{ }^{1} \mathrm{H}-\mathrm{NMR}$ $\left(300 \mathrm{MHz}, \mathrm{CDCl}_{3}, \delta\right): 7.01$ (s, 1H, Ar-H), 6.94 (s, $1 \mathrm{H}, \mathrm{Ar}-\mathrm{H}), 4.81\left(\mathrm{~m}, 1 \mathrm{H}, \mathrm{H}_{5}\right), 4.75-4.71\left(\mathrm{~m}, 2 \mathrm{H}, \mathrm{H}_{3}\right.$, $\left.\mathrm{H}_{4}\right), 4.63$ (s, $\left.4 \mathrm{H}, \mathrm{CH}_{2}-\mathrm{Cl}\right), 4.08-3.87\left(\mathrm{~m}, 8 \mathrm{H}, \mathrm{H}_{1}^{\mathrm{a}}\right.$, $\left.\mathrm{H}_{1}{ }^{\mathrm{b}}, \mathrm{H}_{6}{ }^{\mathrm{a}}, \mathrm{H}_{6}{ }^{\mathrm{b}}, \mathrm{H}_{2}, \mathrm{OCH}_{3}\right), 3.55\left(\mathrm{td},{ }^{4} \mathrm{~J}=5.4 \mathrm{~Hz},{ }^{3} \mathrm{~J}=\right.$ $\left.8.7 \mathrm{~Hz}, 2 \mathrm{H}, \mathrm{OCH}_{2}\right), 1.60\left(\mathrm{~m}, 2 \mathrm{H}, \mathrm{OCH}_{2}-\mathrm{CH}_{2}-\mathrm{CH}_{2}\right.$ ), $1.39-1.30\left(\mathrm{~m}, 6 \mathrm{H},-\left(\mathrm{CH}_{2}\right)_{3}-\mathrm{CH}_{3}\right), 0.91(\mathrm{t}, \mathrm{J}=7.8 \mathrm{~Hz}$, $\left.3 \mathrm{H},-\mathrm{CH}_{3}\right) ;{ }^{13} \mathrm{C}-\mathrm{NMR}\left(75 \mathrm{MHz}, \mathrm{CDCl}_{3}, \delta\right): 152.02$, $148.59,128.18,127.38,115.79,113.66,86.05,85.47$, $83.63,82.49,72.71,72.26,70.18,56.41,41.23$, 31.80, 29.90, 25.93, 22.76, 14.19; FTIR $\left(\mathrm{cm}^{-1}\right): 672$ (s, C-Cl stretching).

e3: $\mathrm{R}$ = dodecyl; recrystallization from chloroform/ ethanol (1:5 v/v); aspect: white solid; yield: $75 \%$; $\mathrm{mp}$ : $86^{\circ} \mathrm{C} ;[\alpha]_{\mathrm{D}}=49^{\circ}\left(2, \mathrm{CHCl}_{3}\right) ;{ }^{1} \mathrm{H}-\mathrm{NMR}(300 \mathrm{MHz}$, $\left.\mathrm{CDCl}_{3}, \delta\right): 7.01$ (s, 1H, Ar-H), $6.94(\mathrm{~s}, 1 \mathrm{H}, \mathrm{Ar}-\mathrm{H})$, $4.82\left(\mathrm{~m}, 1 \mathrm{H}, \mathrm{H}_{5}\right), 4.73-4.71\left(\mathrm{~m}, 2 \mathrm{H}, \mathrm{H}_{3}, \mathrm{H}_{4}\right), 4.64$ (s, 
$\left.4 \mathrm{H},-\mathrm{CH}_{2} \mathrm{Cl}\right), 4.06-3.91\left(\mathrm{~m}, 5 \mathrm{H}, \mathrm{H}_{1}{ }^{\mathrm{a}}, \mathrm{H}_{1}{ }^{\mathrm{b}}, \mathrm{H}_{6}{ }^{\mathrm{a}}, \mathrm{H}_{6}{ }^{\mathrm{b}}\right.$, $\left.\mathrm{H}_{2}\right), 3.88\left(\mathrm{~s}, 3 \mathrm{H}, \mathrm{OCH}_{3}\right), 3.54\left(\mathrm{td},{ }^{4} \mathrm{~J}=5.4 \mathrm{~Hz},{ }^{3} \mathrm{~J}=\right.$ $\left.8.7 \mathrm{~Hz}, 2 \mathrm{H}, \mathrm{OCH}_{2}\right), 1.57\left(\mathrm{~m}, 2 \mathrm{H}, \mathrm{OCH}_{2}-\mathrm{CH}_{2}-\mathrm{CH}_{2}\right)$, $1.27\left(\mathrm{~m}, 18 \mathrm{H},-\left(\mathrm{CH}_{2}\right)_{9}-\mathrm{CH}_{3}\right), 0.92(\mathrm{t}, \mathrm{J}=6 \mathrm{~Hz}, 3 \mathrm{H}$, $\left.\mathrm{CH}_{3}\right) ;{ }^{13} \mathrm{C}$-NMR $\left(75.5 \mathrm{MHz}, \mathrm{CDCl}_{3}, \delta\right): 151.83$, 148.39, 127.98, 127.18, 115.58, 113.44, 85.86, 85.27, $83.42,82.28,72.51,72.06,70,56.21,41.05,41.01$, $31.90,29.74,29.63,29.61,29.58,29.55,29.42$, 29.33, 26.07, 22.67, 14.09; FTIR $\left(\mathrm{cm}^{-1}\right)$ : 671 (s, C-Cl stretching).

\subsection{Synthesis of the polymers (P1-3)}

The corresponding dichloromethyl derivative (e1-3) $(5 \mathrm{mmol})$ was dissolved in DMF $(50 \mathrm{~mL})$ under argon at room temperature. To the obtained solution was slowly added $27 \mathrm{~mL}$ of potassium tert-butoxide $(0.75 \mathrm{M}$, in $t$-butanol). After $40 \mathrm{~h}$, a second fraction $(13.5 \mathrm{~mL})$ of the base was gradually added while heating to $100^{\circ} \mathrm{C}$. After $10 \mathrm{~h}$, the resulting reaction mixture was cooled and $50 \mathrm{~mL}$ of methanol was added. The obtained precipitate was then filtered and dried under vacuum. Further purification of the polymer was carried out by two precipitations in methanol from chloroform solution.

P1: R = ethyl; aspect: red powder; yield: $50 \%$; $[\alpha]_{\mathrm{D}}=$ $-2850^{\circ}\left(0.23, \mathrm{CHCl}_{3}\right) ;{ }^{1} \mathrm{H}-\mathrm{NMR}\left(300 \mathrm{MHz}, \mathrm{CDCl}_{3}\right.$,

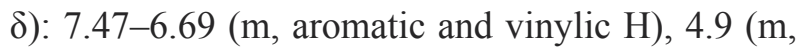
$\mathrm{H}_{5}, \mathrm{H}_{4}, \mathrm{H}_{3}$ ), 4.15-3.97 (m, $\mathrm{H}_{1}{ }^{\mathrm{a}}, \mathrm{H}_{1}{ }^{\mathrm{b}}, \mathrm{H}_{6}{ }^{\mathrm{a}}, \mathrm{H}_{6}{ }^{\mathrm{b}}, \mathrm{H}_{2}$, $\left.\mathrm{OCH}_{3}\right), 3.59\left(\mathrm{~m}, \mathrm{OCH}_{2}\right), 1.23\left(\mathrm{~m}, \mathrm{CH}_{3}\right) ;{ }^{13} \mathrm{C}-\mathrm{NMR}$ $\left(75.5 \mathrm{MHz}, \mathrm{CDCl}_{3}, \delta\right): 152.36,148.93,127.42$, 124.20, 123.47, 112.98, 109.23, 85.95, 85.66, 83.45, 77, 72.45, 71.93, 65.13, 56.32, 15.31; FTIR $\left(\mathrm{cm}^{-1}\right)$ : 3062 (w, aromatic and vinylic C-H stretching), 2938, 2867 (w, aliphatic C-H stretching), 1650, 1560, 1505 ( $\mathrm{m}, \mathrm{C}=\mathrm{C}$ stretching), 1243, 1198 (s, C-O-C asymmetric stretching), $1070(\mathrm{~m}, \mathrm{C}-\mathrm{O}-\mathrm{C}$ symmetric stretching), 962 (m, $E-\mathrm{HC}=\mathrm{CH}$ out-of-plane bending), 790 (s, aromatic C-H out-of-plane bending).

P2: $\mathrm{R}=$ hexyl; aspect: red powder; yield: $51 \% ;[\alpha]_{\mathrm{D}}=$ $-3000^{\circ}\left(0.15, \mathrm{CHCl}_{3}\right) ;{ }^{1} \mathrm{H}-\mathrm{NMR}\left(300 \mathrm{MHz}, \mathrm{CDCl}_{3}\right.$,

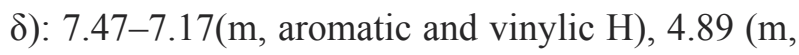
$\left.\mathrm{H}_{5}, \mathrm{H}_{4}, \mathrm{H}_{3}\right), 4.15-3.96\left(\mathrm{~m}, \mathrm{H}_{1}{ }^{\mathrm{a}}, \mathrm{H}_{1}{ }^{\mathrm{b}}, \mathrm{H}_{6}{ }^{\mathrm{a}}, \mathrm{H}_{6}{ }^{\mathrm{b}}, \mathrm{H}_{2}\right.$, $\left.\mathrm{OCH}_{3}\right), 3.54-3.50\left(\mathrm{~m}, \mathrm{OCH}_{2}\right), 1.58-1.31(\mathrm{~m}$, $\left.-\left(\mathrm{CH}_{2}\right)_{3}-\mathrm{CH}_{2}\right), 0.9\left(\mathrm{~m}, \mathrm{CH}_{3}\right) ;{ }^{13} \mathrm{C}-\mathrm{NMR}(75.5 \mathrm{MHz}$, $\left.\mathrm{CDCl}_{3}, \delta\right): 152.46,149,123.97,85.93,85.67,83.62$, 77.42, 72.43, 71.98, 69.94, 56.28, 31.91, 29.76, 25.75, 22.67, 14.18; FTIR $\left(\mathrm{cm}^{-1}\right): 3061$ (w, aromatic and vinylic C-H stretching), 2920, 2856 (w, aliphatic C-H stretching), 1510, 1467, 1412 (m, C=C stretching), 1254, 1205 (s, C-O-C asymmetric stretching),
1093 (m, C-O-C symmetric stretching), 964 (m, $E-\mathrm{HC}=\mathrm{CH}$ out-of-plane bending), 783 (s, aromatic $\mathrm{C}-\mathrm{H}$ out-of-plane bending).

P3: $\mathrm{R}=$ dodecyl; aspect: red powder; yield: $55 \%$; $[\alpha]_{\mathrm{D}}=-3500^{\circ}\left(0.12, \mathrm{CHCl}_{3}\right) ;{ }^{1} \mathrm{H}-\mathrm{NMR}(300 \mathrm{MHz}$, $\left.\mathrm{CDCl}_{3}, \delta\right)$ : 7.39-7.07 (m, aromatic and vinylic $\left.\mathrm{H}\right)$, $4.81\left(\mathrm{~m}, \mathrm{H}_{5}, \mathrm{H}_{4}, \mathrm{H}_{3}\right), 3.97-3.9\left(\mathrm{~m}_{1} \mathrm{H}_{1}^{\mathrm{a}}, \mathrm{H}_{1}{ }^{\mathrm{b}}, \mathrm{H}_{6}{ }^{\mathrm{a}}, \mathrm{H}_{6}{ }^{\mathrm{b}}\right.$, $\left.\mathrm{H}_{2}, \mathrm{OCH}_{3}\right), 3.49-3.42\left(\mathrm{~m}, \mathrm{OCH}_{2}\right), 1.53-1.09$ (m, $\left.\left(\mathrm{CH}_{2}\right)_{10}\right), 0.81-0.76\left(\mathrm{~m}, \mathrm{CH}_{3}\right) ;{ }^{13} \mathrm{C}-\mathrm{NMR}(75.5 \mathrm{MHz}$, $\left.\mathrm{CDCl}_{3}, \delta\right): 152.36,148.78,123.57,113.02,109.02$, $85.95,85.59,83.63,76.57,72.43,72,69.95,56.27$, $31.90,29.78,29.62,29.47,29.33,26.09,22.67$, 14.09; FTIR $\left(\mathrm{cm}^{-1}\right)$ : 3061 (w, aromatic and vinylic $\mathrm{C}-\mathrm{H}$ stretching), 2920, 2856 (w, aliphatic $\mathrm{C}-\mathrm{H}$ stretching), 1595, 1510, 1460 ( $\mathrm{m}, \mathrm{C}=\mathrm{C}$ stretching), 1254, 1205 (s, C-O-C asymmetric stretching), 1084 (m, C-O-C symmetric stretching), 964 (m, $E-\mathrm{CH}=\mathrm{CH}$ out-of-plane bending), 783 (s, aromatic $\mathrm{C}-\mathrm{H}$ out-of-plane bending).

\subsection{Fabrication and characterization of the diodes}

Single-layer devices were elaborated as sandwich structures between an aluminium $(\mathrm{Al})$ cathode and an indium tin oxide (ITO) (ITO-thickness of $100 \mathrm{~nm}$, sheet resistance of $20 \Omega /$ square) anode. Polymer solution $\left(2 \cdot 10^{-2} \mathrm{M}\right.$ in chloroform) was spin-cast (2500 rpm) onto ITO glass to obtain a film about $60 \mathrm{~nm}$ thick after annealing at $40^{\circ} \mathrm{C}$ for $1 \mathrm{~h}$. A thin aluminium layer $(150 \mathrm{~nm})$ was deposited by thermal evaporation at $3 \cdot 10^{-6}$ Torr. The currentvoltage (I-V) characteristics of the devices were recorded with a Keithley 236 source meter (Keithley, Les Ulis, France).

\section{Results and discussion \\ 3.1. Synthesis and characterization}

New isosorbide-containing chiral monomers (e1-3) were synthesized following a five-step pathway (Figure 1). The 2,5-O-ditosyl-1,4:3,6-dianhydro-Dsorbitol (a) was prepared in THF/aqueous $\mathrm{NaOH}$ two-phase system, according a previously described procedure [27]. The tosylated isosorbide was treated with methoxyphenol in sodium carbonate/DMF system; thereby, a selective endo-monoalkylated isosorbide derivative (b) was obtained. Indeed, the exo position reactivity is limited by the steric hindrance effect [28]. The deprotection of the isosorbide exo hydroxyl group was carried out by sodium ethoxide in ethanol [29]. The obtained derivative 


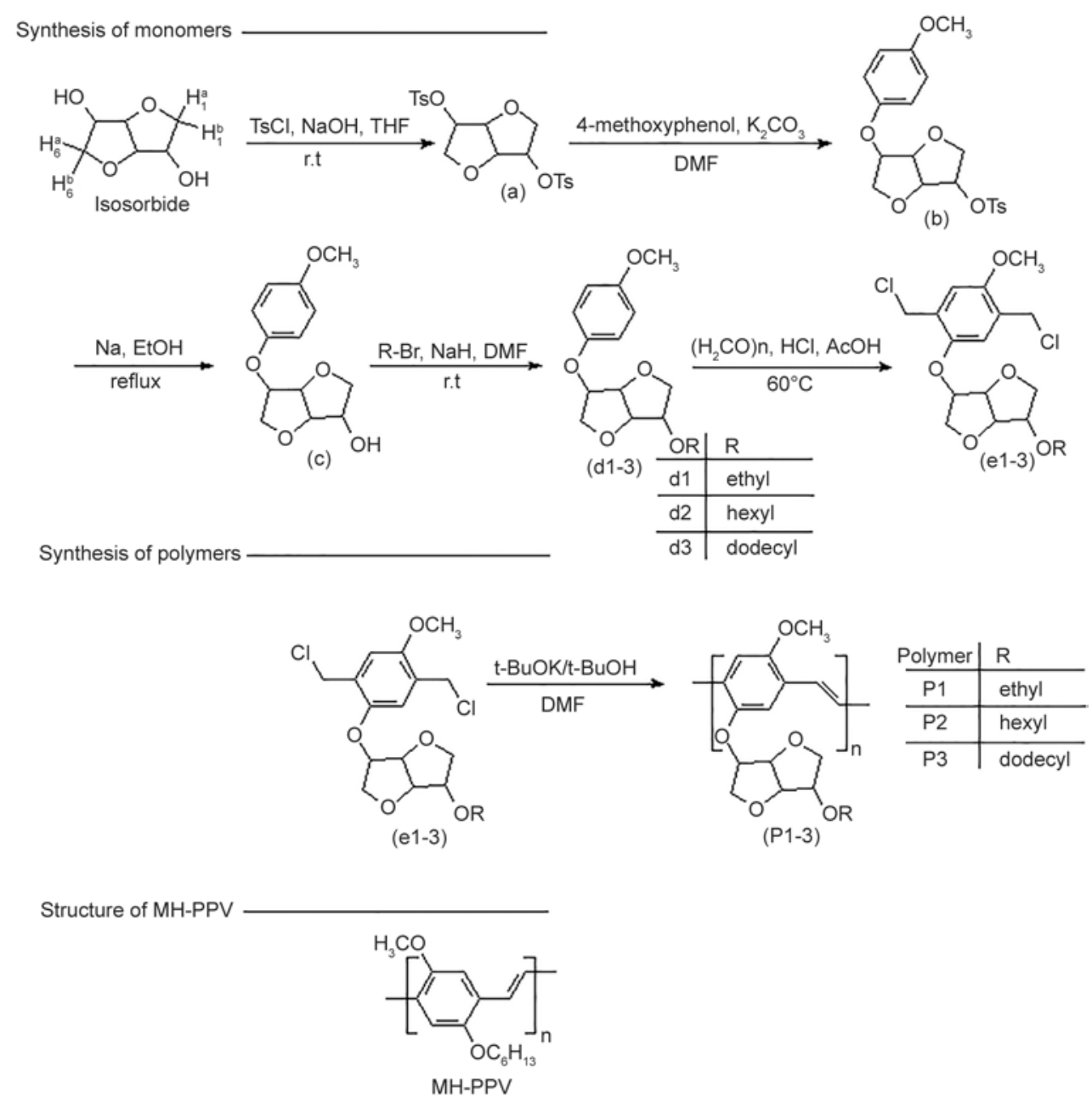

Figure 1. Synthetic route to the monomers and the polymers and macromolecular structure of MH-PPV

(c) was then O-alkylated with different aliphatic chain: ethyl, hexyl and dodecyl. The aromatic group of the isosorbide diethers (d1-3) was chloromethylated using the $\mathrm{HCl} /$ paraformaldehyde/acetic acid system [30] to obtain the corresponding isosorbide-containing monomers (e1-3). The PPV polymers (P1-3) were synthesized via the Gilch polycondensation of these aromatic dichloromethyl derivatives in DMF and using tert-butoxide as the basic condensing agent [20] (Figure 1). The PPV derivatives are optically active with a specific optical rotation $\left([\alpha]_{D}\right)$ of $-2850,-3000$ and $-3500^{\circ}$ for P1, P2 and P3, respectively. The polymers were found to have good solubility in common organic solvents such as chloroform, dichloromethane, THF and toluene. The macromolecular structures were confirmed by NMR and FTIR spectroscopic analyses. The ${ }^{1} \mathrm{H}-\mathrm{NMR}$ spectra (Figure 2 ) show a broad peak between 7.4 and $7.1 \mathrm{ppm}$, wich was assigned to the aromatic and vinylic protons. The $\mathrm{CH}_{2} \mathrm{O}$, $\mathrm{CH}_{3} \mathrm{O}$ groups and dianhydro protons appear at the 4.9-3.3 ppm range and the aliphatic groups give rise to a multiplet between 1.6 and $1.1 \mathrm{ppm}$. For all polymers, the IR spectra (Figure 3) reveal an absorption band centered at $960 \mathrm{~cm}^{-1}$ which is assigned to the out-of-plane vibration of the $E$-vinylene groups. The $Z$-vinylene characteristic band (about $860 \mathrm{~cm}^{-1}$ ) $[19,31]$ was not observed, indicating a dominant $E$ configuration. The number-average molecular weights $\left(M_{\mathrm{n}}\right)$ were in the range of 16000 $21000 \mathrm{~g} \cdot \mathrm{mol}^{-1}$ and the polydispersity indices $\left(I_{\mathrm{p}}\right)$ were between 2.6 and 2.9, as determined by SEC analysis (Table 1). The thermal properties of P1-3 were investigated by thermogravimetric (TGA) and differential scanning calorimetry (DSC) under ambient atmospheric conditions. The thermograms indicate that the polymers begin to degrade at about $320^{\circ} \mathrm{C}$, losing less than $5 \%$ of their weight (Figure 4). The major degradation takes place at $375^{\circ} \mathrm{C}$; this process corresponds to the decomposition of the side-groups. The P1-3 showed improved thermal stability, in comparison with the PPV derivatives containing aliphatic side-chains, which decompose below $300^{\circ} \mathrm{C}$ [32]. This behavior is due to the incor- 

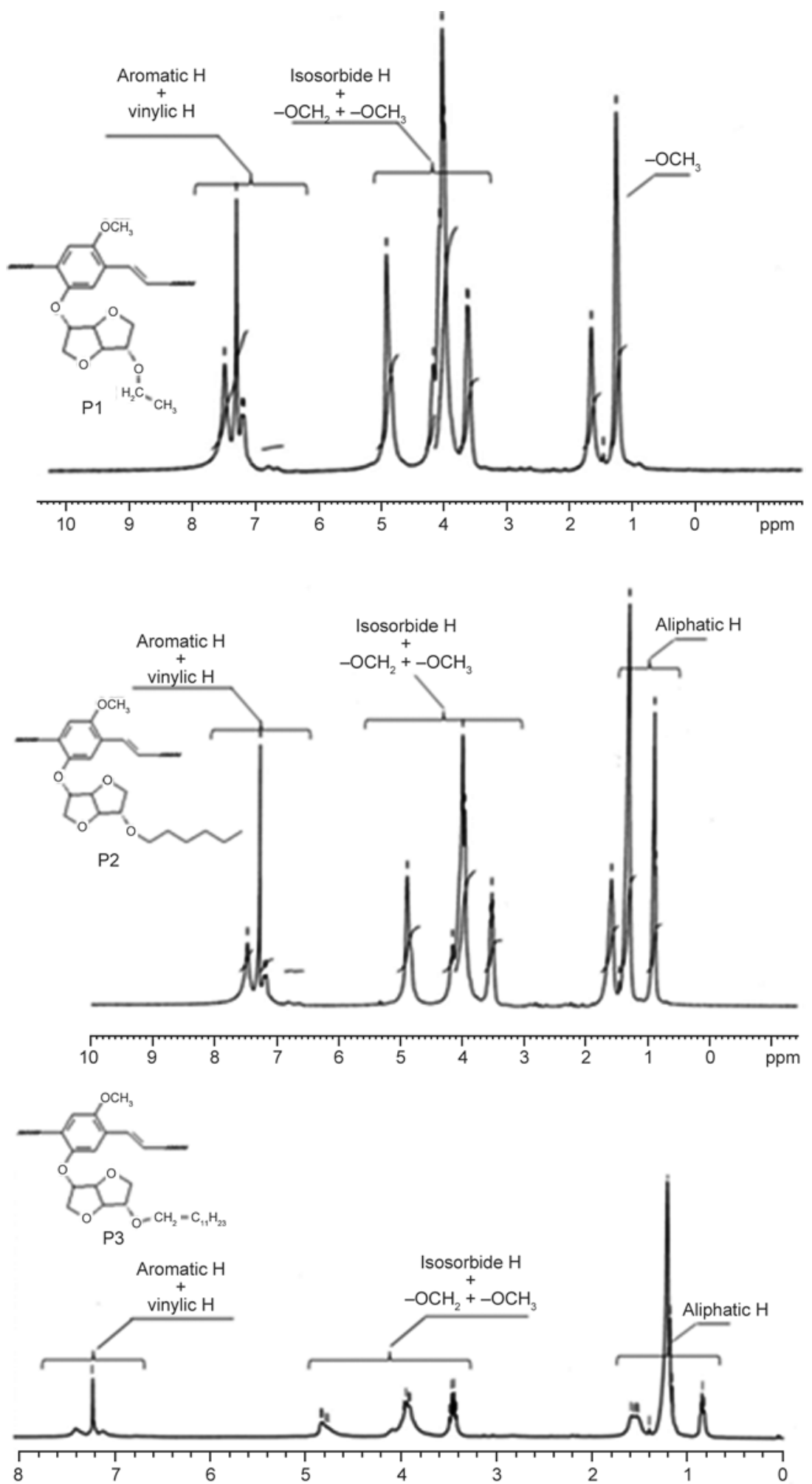

Figure 2. ${ }^{1} \mathrm{H}-\mathrm{NMR}$ spectra of $\mathbf{P 1 - 3}$

Table 1. SEC characterizations

\begin{tabular}{|l|c|c|c|c|}
\hline Polymer & $\overline{\mathbf{M}}_{\mathbf{n}}^{\left({ }^{(a)}\right.}$ & $\overline{\mathbf{M}}_{\mathbf{w}}{ }^{(\mathbf{b})}$ & $\mathbf{I}_{\mathbf{p}}{ }^{(\mathbf{c})}$ & $\overline{\mathbf{D P}}_{\mathbf{n}}{ }^{(\mathbf{d})}$ \\
\hline P1 & 16420 & 47770 & 2.91 & 54 \\
\hline $\mathbf{P 2}$ & 21240 & 56290 & 2.65 & 59 \\
\hline $\mathbf{P 3}$ & 17760 & 46530 & 2.62 & 40 \\
\hline
\end{tabular}

(a) number average molecular weight; ${ }^{(b)}$ weight average molecular weight; ${ }^{(c)}$ polydispersity indices; ${ }^{(d)}$ number average degree of polymerization. poration of the rigid and polar isosorbide units in the side-chain. The DSC results indicate a glass transition temperature $\left(T_{\mathrm{g}}\right)$ about $75^{\circ} \mathrm{C}$ for $\mathbf{P 1}, 50^{\circ} \mathrm{C}$ for $\mathbf{P} 2$ and $100^{\circ} \mathrm{C}$ for $\mathbf{P 3}$. No melting or other thermal events were observed, which suggested that the polymers were completely amorphous. 


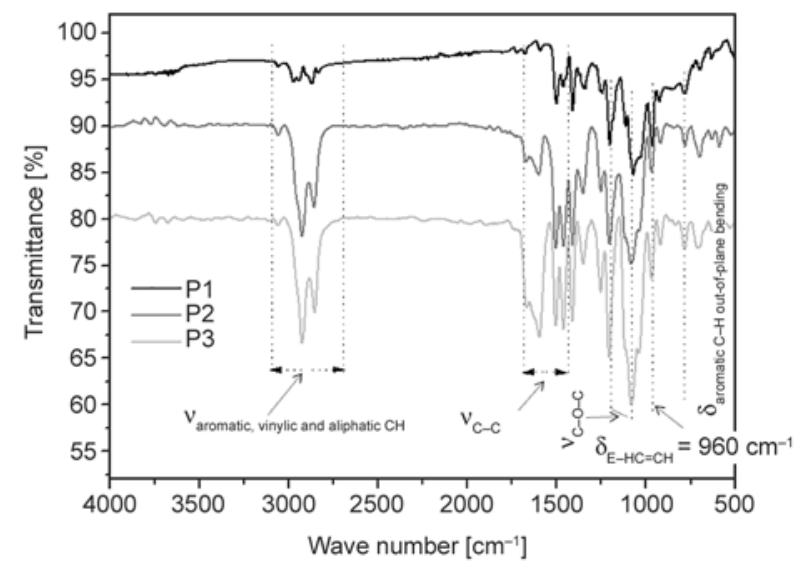

Figure 3. IR spectra of P1-3

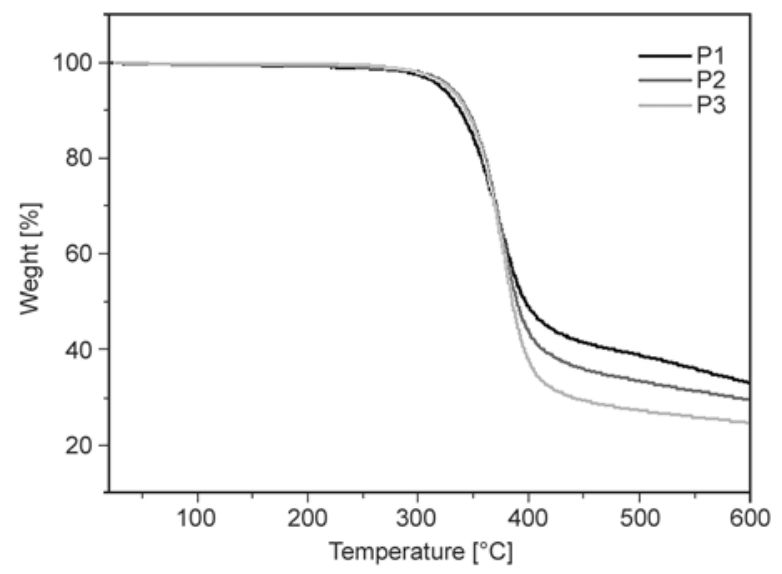

Figure 4. TGA thermograms of P1-3 (under nitrogen; heating rate: $10^{\circ} \mathrm{C} \cdot \mathrm{min}^{-1}$ )

\subsection{Thin-film surface properties}

Polymer films on a glass substrate were prepared and characterized by atomic force microscopy (AFM) (Figure 5). The results show a smooth surface with a root-mean-square (RMS) of $2.5 \mathrm{~nm}$ for the dodecyloxy-containing polymer (P3). The surface roughness increases with decreasing side chain-
Table 2. Surface characteristics of untreated and P1-3 treated glass

\begin{tabular}{|c|c|c|c|c|c|}
\hline & $\boldsymbol{\gamma}^{+}$ & $\boldsymbol{\gamma}^{-}$ & $\boldsymbol{\gamma}^{\mathbf{A B}}$ & $\boldsymbol{\gamma}^{\mathbf{L W}}$ & $\boldsymbol{\gamma}^{\mathbf{S}}$ \\
\hline Glass surface & 2.0 & 47.8 & 19.5 & 36.7 & 56.1 \\
\hline P1 & 0.7 & 44.6 & 11.2 & 42.1 & 53.6 \\
\hline P2 & 1.0 & 26.6 & 10.3 & 47.6 & 57.7 \\
\hline P3 & 1.8 & 45.0 & 18.0 & 34.1 & 52.1 \\
\hline
\end{tabular}

$\gamma^{+}$: acid energy component; $\gamma^{-}$: basic energy component $; \gamma^{\mathrm{AB}}=$ $2\left(\gamma^{+} \gamma^{-}\right)^{1 / 2}$ : polar energy; $\gamma^{\mathrm{LW}}$ : apolar energy; $\gamma^{\mathrm{S}}=\gamma^{\mathrm{AB}}+\gamma^{\mathrm{LW}}$ : surface energy.

length: RMS values of 4.3 and $7.1 \mathrm{~nm}$ were found for $\mathbf{P} 2$ and $\mathbf{P 1}$, respectively. The thin films were also investigated by contact angle measurements, which provide a convenient way to characterize the surface polarity and reflect its functionality [33]. The van Oss-Chaudhury-Good model was exploited, using three test liquids (water, diiodomethane and formamide) [34].

For all polymers, the basic energy component outweighs the acidic aspect, which is due to the presence of oxygen-rich isosorbide units. However, higher polar energy was obtained in the case of $\mathbf{P 3}$ film, indicating a higher isosorbide density in its surface (Table 2).

\subsection{Optical properties}

The optical absorption and photoluminescence (PL) properties of the polymers were investigated both for chloroform solutions and thin solid films. The concentrations of the chloroform solutions were fixed at $5 \cdot 10^{-5} \mathrm{M}$ in absorption and $2 \cdot 10^{-7} \mathrm{M}$ and in PL. Uniform polymer films were prepared on quartz plates by spin-coating from chloroform solution at room temperature. The absorption and emission data for the polymers are summarized in Table 3.

Table 3. Optical data for P1-3

\begin{tabular}{|c|c|c|c|c|c|c|c|}
\hline \multicolumn{8}{|c|}{ Dilute solution in chloroform } \\
\hline \multicolumn{5}{|c|}{ Absorption } & \multicolumn{3}{|c|}{ Photoluminescence } \\
\hline & $\begin{array}{l}\lambda_{\max } \\
{[\mathrm{nm}]}\end{array}$ & $\begin{array}{c}\varepsilon_{\max } \\
{\left[10^{4} \cdot \mathbf{M}^{-1} \cdot \mathbf{c m}^{-1}\right]}\end{array}$ & $\begin{array}{l}\text { FWHM }^{(\mathbf{b})} \\
{[\mathrm{nm}]}\end{array}$ & $\begin{array}{l}\lambda_{\text {onset }} \\
{[\mathrm{nm}]}\end{array}$ & \multicolumn{2}{|c|}{$\begin{array}{l}\lambda_{\max } \\
{[\mathrm{nm}]}\end{array}$} & $\Phi_{\mathrm{fl}}{ }^{(c)}$ \\
\hline P1 & 480 & 2.38 & 97 & 544 & \multicolumn{2}{|c|}{$543 ; 582^{(a)}$} & 0.51 \\
\hline $\mathbf{P 2}$ & 480 & 1.93 & 96 & 542 & \multicolumn{2}{|c|}{$540 ; 583^{(\mathrm{a})}$} & 0.75 \\
\hline $\mathbf{P 3}$ & 470 & 1.93 & 109 & 542 & \multicolumn{2}{|c|}{$543 ; 582^{(a)}$} & 0.73 \\
\hline \multicolumn{8}{|c|}{ Thin film } \\
\hline \multicolumn{5}{|c|}{ Absorption } & \multicolumn{3}{|c|}{ Photoluminescence } \\
\hline & $\begin{array}{l}\lambda_{\max } \\
{[\mathrm{nm}]}\end{array}$ & $\begin{array}{l}\lambda_{\text {onset }} \\
{[\mathrm{nm}]}\end{array}$ & \multicolumn{2}{|c|}{$\begin{array}{c}\text { FWHM }^{(\mathbf{b})} \\
{[\mathrm{nm}]}\end{array}$} & & $\begin{array}{c}\text { FWHM }^{(\mathbf{b})} \\
{[\mathrm{nm}]}\end{array}$ & $\Phi_{\mathrm{fl}}^{(\mathrm{c})}(\mathrm{Pi}) / \Phi_{\mathrm{fl}}^{(\mathrm{c})}(\mathrm{P} 1)$ \\
\hline P1 & 485 & 585 & \multicolumn{2}{|c|}{136} & & 113 & 1.0 \\
\hline $\mathbf{P 2}$ & 488 & 593 & \multicolumn{2}{|c|}{161} & $99^{(a)}$ & 115 & 1.3 \\
\hline $\mathbf{P 3}$ & 479 & 585 & \multicolumn{2}{|c|}{138} & $7^{(\mathrm{a})}$ & 116 & 1.2 \\
\hline
\end{tabular}

${ }^{\text {(a) Shoulder, }}{ }^{(b)}$ Spectrum full width at half maximum, ${ }^{(c)}$ PL quantum yields 

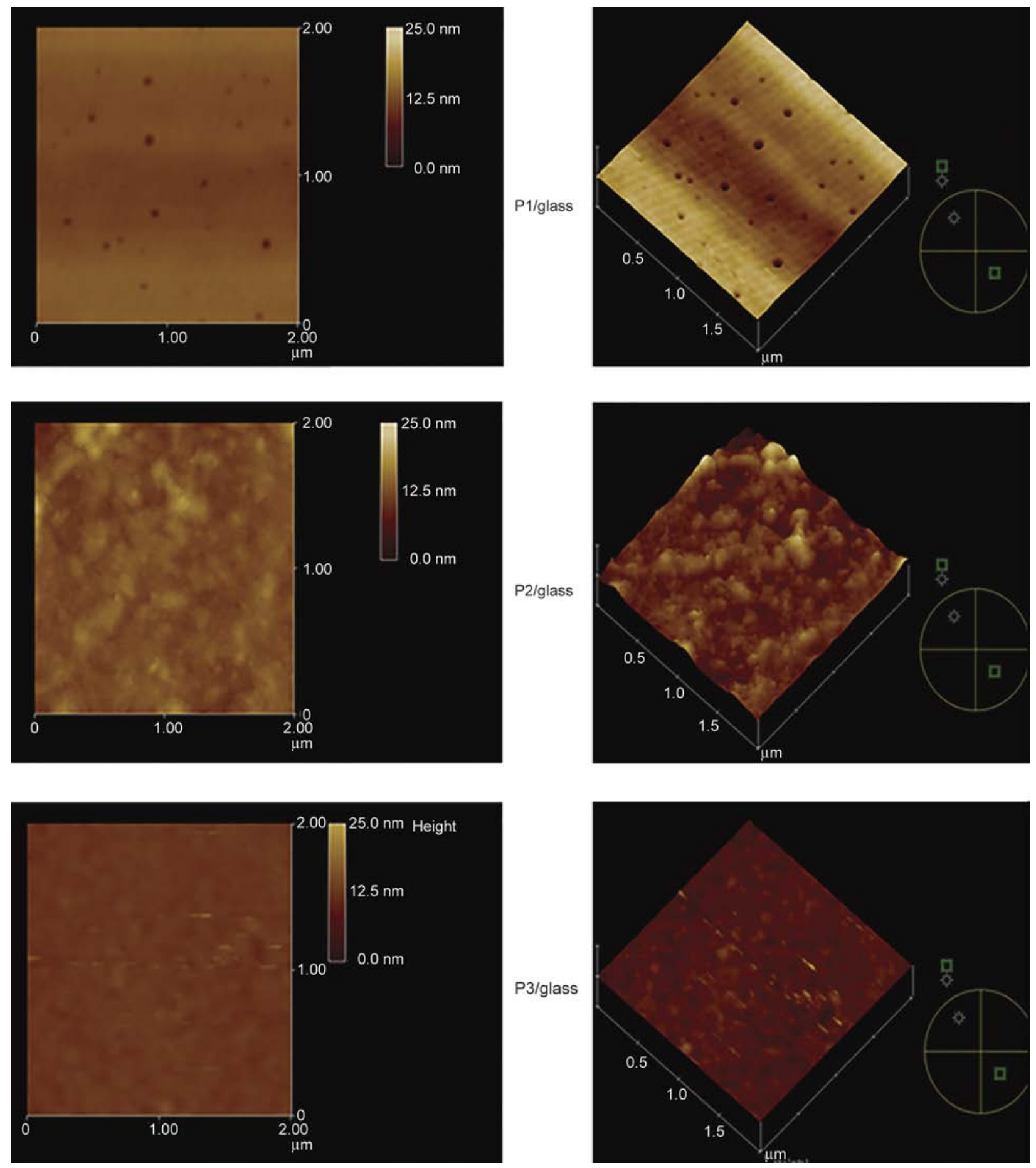

Figure 5. AFM images of [glass/polymer] layers

The absorption and PL spectra of P1-3 solutions are illustrated in Figure 6. The UV-vis spectra show a similar form with a maximum absorption at $480 \mathrm{~nm}$ for $\mathbf{P 1}$ and $\mathbf{P 2}$ and at $470 \mathrm{~nm}$ for P3. The different behaviors can be explained by the change in the environment surrounding the PPV conjugate systems. Indeed, because $\mathbf{P} \mathbf{3}$ has relatively long dodecyloxy side-chains, the environment of the $\pi$-conjugate system is less polar, therefore, its UV-vis absorption is blue-shifted compared to $\mathbf{P 1}$ and $\mathbf{P 2}$. On the other hand, the spectra of the polymer solutions show the same absorption onset, indicating no effect of the side-group size on the effective conjugation length. The comparison of $\mathbf{P 3}$ with the previously reported PPV derivative containing dodecyloxy side-chain [20] indicates a slight hypsochromic effect $(\sim 10 \mathrm{~nm})$. Such behavior is probably due to the steric hindrance of the isosorbide groups, which decrease the planarity of the conjugated system, therefore, reduce the effective conjugation length. The polymer solu- 


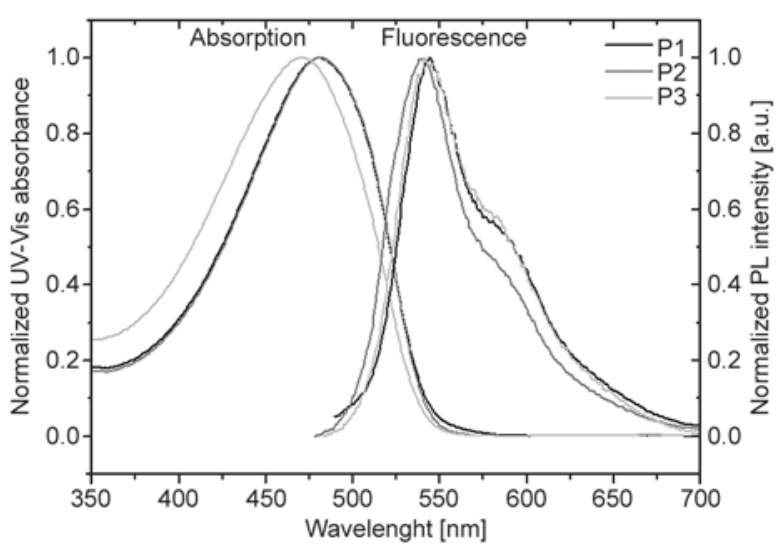

Figure 6. UV-vis absorption and PL spectra of polymers in chloroform $\left(5 \cdot 10^{-5} \mathrm{M}\right.$ in absorption and $2 \cdot 10^{-7} \mathrm{M}$ in PL)

tions exhibit a yellow fluorescence with a main emission band at $540 \mathrm{~nm}$ and a shoulder around $580 \mathrm{~nm}$. Such vibrational structure is characteristic of the PPV system and is attributed to a coupling of the phenylene ring stretching modes with the $\pi-\pi^{*}$ electronic transitions [35]. The fluorescence quantum efficiencies of the isosorbide-based polymers were determined in dilute chloroform by a relative method using quinine sulfate as standard [26]. The obtained values are 51, 75 and $73 \%$ for P1, P2 and $\mathbf{P 3}$, respectively. The polymer $\mathbf{P} 2$ exhibits a significantly enhanced PL intensity in comparison with its isosorbide-free analogue MH-PPV (Figure 1), which shows a PL efficiency of $36 \%$. This behavior can be attributed to the presence of the isosorbide moieties, which increase the rigidity of the macromolecular architecture. Besides, these polar groups may generate Keesom-type intramolecular interactions, which block the conformational changes. Hence, the available vibrational and rotational degrees of freedom were reduced and the loss of PL by such processes was considerably limited $[36,37]$.

The absorption and PL spectra of a solid film of P1-3 are displayed in Figure 7. The polymer films present qualitatively the same absorption form as the corresponding solutions. Nevertheless, the spectra are broader and the absorption onsets significantly red-shifted. In fact, similar behavior is generally observed in $\pi$-conjugated polymers, and is attributed to the $\pi-\pi$ interaction of the conjugated systems, hence, aggregate formation in the solid state [38]. The optical band gaps ( $\left.E_{\text {g-op }}\right)$ estimated from the absorption onsets of the polymer films are 2.12, 2.09 and $2.12 \mathrm{eV}$ for $\mathbf{P 1}, \mathbf{P} 2$ and $\mathbf{P 3}$, respectively.

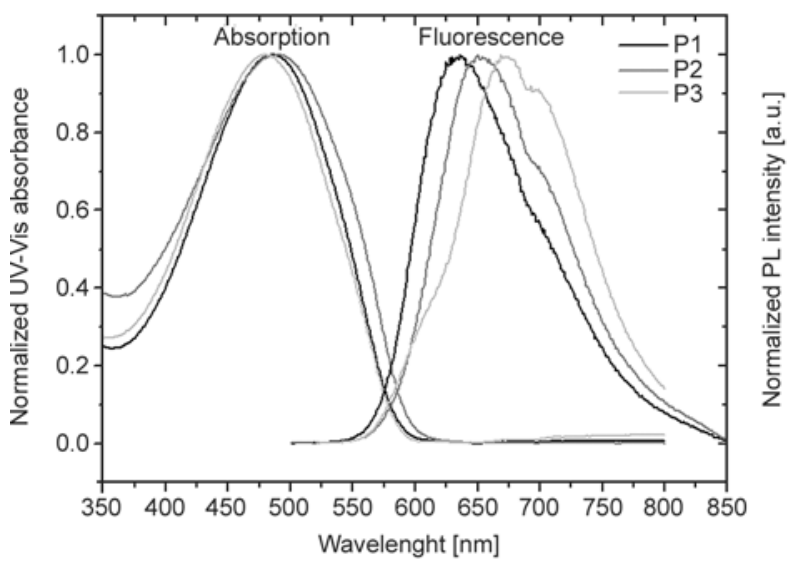

Figure 7. UV-vis absorption and PL spectra of polymers in thin films $(60 \mathrm{~nm})$

The PL spectra of the polymer films show an orange emission with similar features (Figure 7). In comparison with solution state, broader and redshifted spectra were obtained (Table 3), as result of excimer formation [39]. However, the red-shift value increases from $\mathbf{P 1}$ to $\mathbf{P 3}$, suggesting a higher interchain interaction in the $\mathbf{P 3}$ film. This red-shift order is opposite to that obtained in our previously study of the isosorbide-free analogues of P1-3 [20]. The difference can be explained by a more regular supramolecular organization in $\mathbf{P 1 - 3}$, as described in Figure 8 for the $\mathbf{P 2}$ comparing with MH-PPV. According to these suggested arrangements of the PPV systems, the interactions between the conjugated sequences were limited by the aliphatic side-

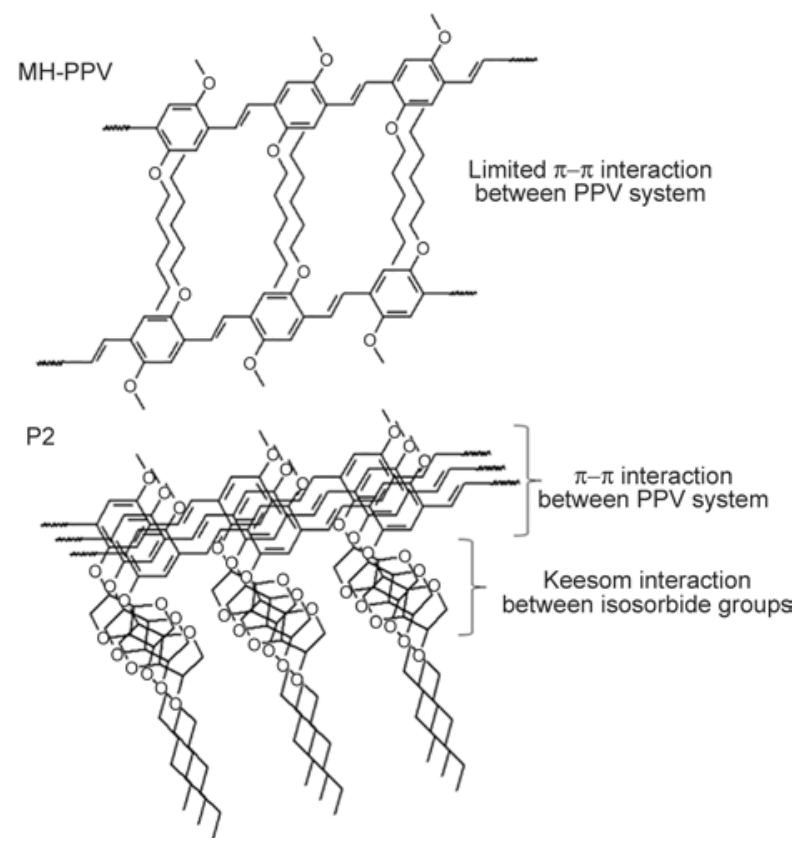

Figure 8. Suggested supramolecular organization of the PPV systems in $\mathbf{P 2}$ and MH-PPV 


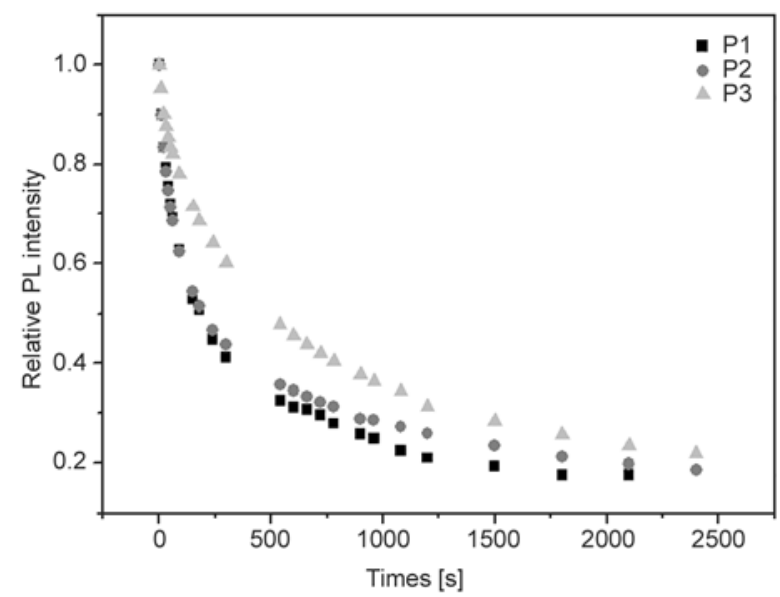

Figure 9. PL decay P1-3 films upon irradiation at $480 \mathrm{~nm}$

chain in MH-PPV. Conversely, in $\mathbf{P 2}$ film, the presence of the polar isosorbide groups improves the inter-chain stacking by the Keesom interaction and changes consequently the probable arrangement of the PPV backbone. In thin solid film, under the identical conditions, the $\mathbf{P 2}$ shows the higher PL quantum yield as supported by the ratios of the polymer PL yields cited in Table 3. The Photobleaching of polymer films was examined under excitation at $480 \mathrm{~nm}$. For all polymers, the emission spectra show no change in shape during irradiation for $40 \mathrm{~nm}$. However, the fluorescence intensity decreases with time as presented in Figure 9. The results show that dodecyloxy containing polymer (P3) has the higher photo-oxidative stability.

\subsection{Electrochemical and electrical characterization}

Cyclic voltammetry (CV) was employed to investigate the redox behavior of the materials and to estimate their HOMO (Highest Occupied Molecular Orbital) and LUMO (Lowest Unoccupied Molecular Orbital) energy levels. Knowledge of these energy levels is of crucial importance to the selection of cathode and anode materials for OLED devices [40]. The use of CV analysis is reliable, as the electrochemical processes are similar to those involved in charge injection and transport processes in OLEDs [41]. The organic films were drop-coated onto an ITO glass substrate and scanned both positively and negatively in $(n-\mathrm{Bu})_{4} \mathrm{NBF}_{4} /$ acetonitrile. The cyclic voltammograms obtained are shown in Figure 10.

According to an empirical method [42] and by assuming that the energy level of the ferrocene/ferroce-

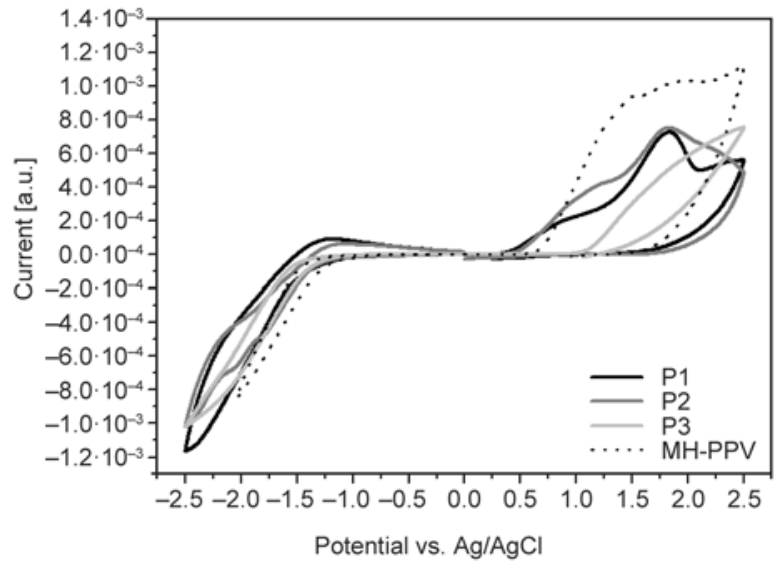

Figure 10. Cyclic voltammograms for polymer films coated onto ITO electrode (in $0.1 \mathrm{M}(\mathrm{n}-\mathrm{Bu})_{4} \mathrm{NBF}_{4} /$ acetonitrile; scan rate: $\left.50 \mathrm{mV} \cdot \mathrm{s}^{-1}\right)$

nium couple is $4.8 \mathrm{~V}$ below the vacuum level, the HOMO energy level $\left(E_{\text {HOMO }}\right)$, LUMO energy level $\left(E_{\mathrm{LUMO}}\right)$ and the electrochemical gap $\left(E_{\mathrm{g} \text {-el }}\right)$ can be calculated as shown by Equations (3)-(5):

$E_{\mathrm{HOMO}}(\mathrm{IP}$, ionization potential $)=$

$$
-\left(V_{\text {onset-ox }}-V_{\mathrm{FOC}}+4.8\right) \mathrm{eV}
$$

$E_{\text {LUMO }}($ EA, electron affinity $)=$

$$
-\left(V_{\text {onset-red }}-V_{\mathrm{FOC}}+4.8\right) \mathrm{eV}
$$

$E_{\mathrm{g}-\mathrm{el}}=\left(E_{\mathrm{LUMO}}-E_{\mathrm{HOMO}}\right) \mathrm{eV}$

where $V_{\mathrm{FOC}}$ is the ferrocene half-wave potential $(0.92 \mathrm{~V}), V_{\text {onset-ox }}$ the polymer oxidation onset and $V_{\text {onset-red }}$ the polymer reduction onset, all measured versus $\mathrm{Ag} / \mathrm{AgCl}$. Accompanying the doping process, there was an obvious color change in the polymer film, i.e. orange in the neutral state and dark brown in the doped state, but no obvious reversible doping and dedoping process was observed. The HOMO and LUMO energy levels were estimated to be 4.27-4.97 and 2.46-2.54 eV, respectively. The electrochemical data-based energy diagram of polymers is presented in Figure 11. Analysis of the energy levels of the three polymers shows comparable electron affinities. However, the ionization potential increases with the side-chain length. The poly (2-hexyloxy-5methoxy- $p$-phenylene vinylene) (MH-PPV) was used to compare the energy levels of HOMO and LUMO with that of its analogue with isosorbide group in side chain. It indicates that the HOMO level of $\mathbf{P 2}$ is $0.2 \mathrm{eV}$ higher in energy than that MH-PPV. Further, both polymers exhibit almost identical electronic affinities; hence, it showed a 


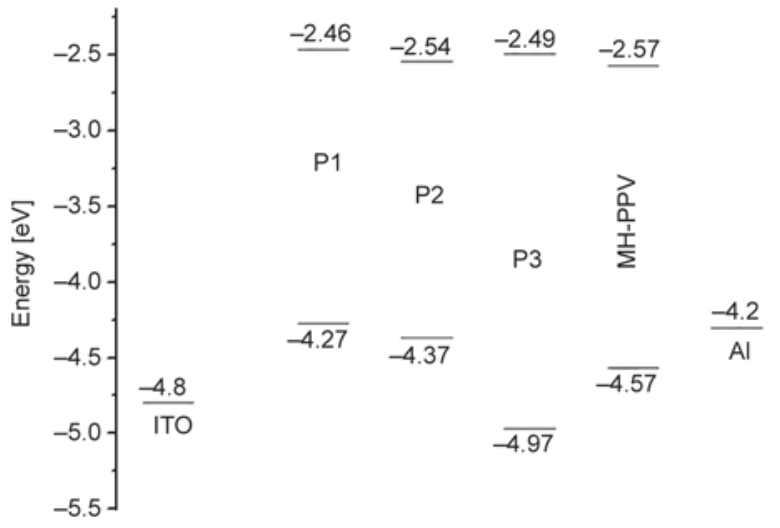

Figure 11. Energy diagram of ITO, P1-3, MH-PPV and Al
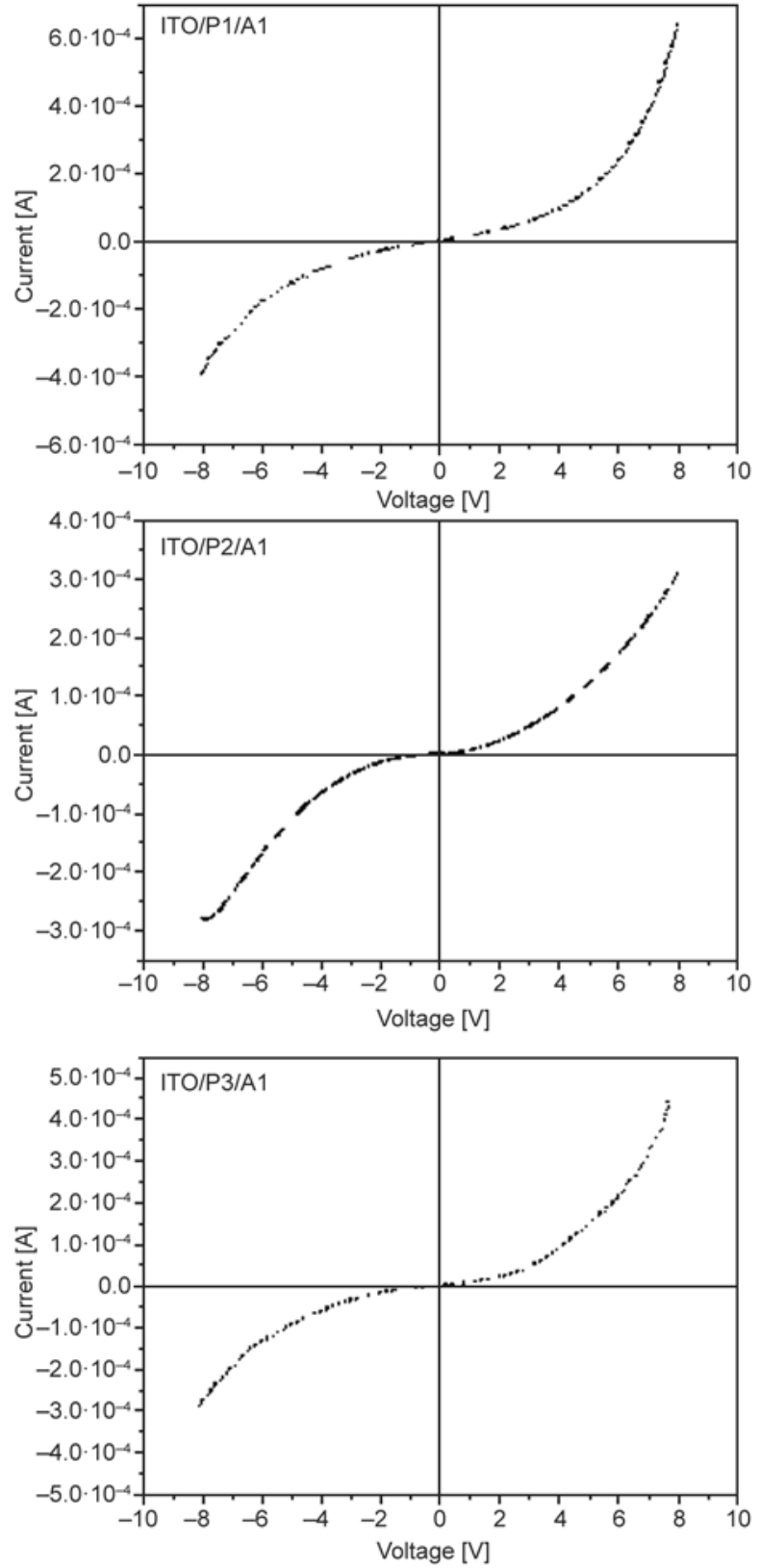

Figure 12. Current-voltage curve for [ITO/polymer/Al] devices lower electrochemical gap. Three single-layer devices with the [ITO/polymer/Al] configuration were fabricated to investigate the current-voltage (I-V) characteristics of the isosorbide-PPV derivatives. As shown in Figure 12, the I-V curves indicate typical diode behavior with relatively low turnon voltages of 3.41, 3.11 and 3.46 V for P1, P2 and P3, respectively.

\section{Conclusions}

We have synthesized and investigated a series of semi-conducting isosorbide-PPVs: various sidechains were attached to the PPV system (ethyloxy: P1, hexyloxy: P2 and dodecyloxy: P3). The polymers are prepared by Gilch polycondensation of chiral monomers. The synthesized polymers are optically active; they have good solubility and thermal stability going up to $320^{\circ} \mathrm{C}$. The best homogeneity and the highest polarity were obtained for the $\mathbf{P 3}$ film. The optical band gaps of the polymer thin films are around $2.1 \mathrm{eV}$. The polymer solutions exhibit a yellow fluorescence $(540 \mathrm{~nm})$ with relatively narrow emission spectra. The fluorescence quantum efficiencies of the isosorbide-containing polymers were determined in dilute solution and were 51,75 and $73 \%$ for $\mathbf{P 1}, \mathbf{P} 2$ and $\mathbf{P 3}$, respectively. The I-V characteristics of the devices with an ITO/polymer/Al configuration demonstrate typical diode behavior with relatively low turn-on voltages.

\section{Acknowledgements}

This work was supported by DGRS of the Ministry of High Education and Research fund of Tunisia. The authors thank Ms Amna Debbebi (Faculty of Sciences, Monastir) for the NMR measurements and Mr Haikel Hrichi (Laboratoire des Interfaces et Matériaux Avancés (LIMA), Faculty of Sciences, Monastir) for the electrical measurements. Special thanks go to John Lomas for his help in improving the quality of the English of this article and for his scientific remarks.

\section{References}

[1] Burroughes J. H., Bradley D. D. C., Brown A. R., Marks R. N., Mackay K., Friend R. H., Burns P. L., Holmes A. B.: Light-emitting diodes based on conjugated polymers. Nature, 347, 539-541 (1990). DOI: $10.1038 / 347539 \mathrm{a} 0$ 
[2] Chen S-H., Li Y-R., Yu C-F., Lin C-F., Kao P-C.: Enhanced luminescence efficiency of $\mathrm{Ag}$ nanoparticles dispersed on indium tin oxide for polymer light-emitting diodes. Optics Express, 21, 26236-26243 (2013). DOI: $10.1364 / \mathrm{OE} .21 .026236$

[3] Kim C-G., Koo M-S., Choi S-H., Lee D-H., Park DK.: Enhanced performance in polymer light-emitting diodes (PLEDs) by using water soluble conjugation polymer. Bulletin of the Korean Chemical Society, 33, 3087-3090 (2012).

DOI: $10.5012 /$ bkcs.2012.33.9.3087

[4] Zheng H., Zheng Y., Liu N., Ai N., Wang Q., Wu S., Zhou J., Hu D., Yu S., Han S., Xu W., Luo C., Meng Y., Jiang Z., Chen Y., Li D., Huang F., Wang J., Peng J., Cao Y.: All-solution processed polymer light-emitting diode displays. Nature Communications, 4, 1-7 (2013). DOI: $10.1038 /$ ncomms2971

[5] Deng X-Y.: Light-emitting devices with conjugated polymers. International Journal of Molecular Sciences, 12, 1575-1594 (2011).

DOI: $10.3390 /$ ijms12031575

[6] Teichler A., Shu Z., Wild A., Bader C., Nowotny J., Kirchner G., Harkema S., Perelaer J., Schubert U. S.: Inkjet printing of chemically tailored light-emitting polymers. European Polymer Journal, 49, 2186-2195 (2013).

DOI: $10.1016 /$ j.eurpolymj.2013.03.031

[7] Cataldo S., Pignataro B.: Polymeric thin films for organic electronics: Properties and adaptive structures. Materials, 6, 1159-1190 (2013).

DOI: $10.3390 / \mathrm{ma} 6031159$

[8] Lee J., Kim D. H., Kim J-Y., Yoo B., Chung J.W., Park J-I., Lee B-L., Jung J. Y., Park J. S., Koo B., Im S., Kim J. W., Song B., Jung M-H., Jang J. E., Jin Y. W., Lee SY.: Reliable and uniform thin-film transistor arrays based on inkjet-printed polymer semiconductors for full color reflective displays. Advanced Materials, 25, 5886-5892 (2013).

DOI: $10.1002 /$ adma.201301257

[9] Kim Y., Lim E.: Development of polymer acceptors for organic photovoltaic cells. Polymers, 6, 382-407 (2014). DOI: $10.3390 /$ polym6020382

[10] Berger R., Domanski A. L., Weber S. A. L.: Electrical characterization of organic solar cell materials based on scanning force microscopy. European Polymer Journal, 49, 1907-1915 (2013).

DOI: $10.1016 /$ j.eurpolymj.2013.03.005

[11] Palaparthy V. S., Baghini M. B., Singh D. N.: Review of polymer-based sensors for agriculture-related applications. Emerging Materials Research, 2, 166-180 (2013).

DOI: $10.1680 / \mathrm{emr} .13 .00010$

[12] Bisri S. Z., Takenobu T., Iwasa Y.: The pursuit of electrically-driven organic semiconductor lasers. Journal of Materials Chemistry C, 2, 2827-2836 (2014).

DOI: $10.1039 / \mathrm{C} 3 \mathrm{TC} 32206 \mathrm{H}$
[13] Chen S. J., Zhang Q. Y., Gu J. W., Ma M. L., Zhang L., Zhou J., Zhou Y. Y.: A new conjugated polymer with donor-acceptor architectures based on alternating 1,4divinyl-2,5-dioctyloxybenzene and 5,8-(2,3-dipyridyl)quinoxaline: Synthesis, characterization, and photoinduced charge transfer. Express Polymer Letters, 6, 454-464 (2012).

DOI: $10.3144 /$ expresspolymlett.2012.48

[14] Botiz I., Schaller R. D., Verduzco R., Darling S. B: Optoelectronic properties and charge transfer in donoracceptor all-conjugated diblock copolymers. The Journal of Physical Chemistry C, 115, 9260-9266 (2011). DOI: $10.1021 / j p 201344 p$

[15] Segura J. L.: The chemistry of electroluminescent organic materials. Acta Polymerica, 49, 319-344 (1998). DOI: 10.1002/(SICI)1521-4044(199807)49:7<319:: AID-APOL319>3.0.CO;2-Q

[16] Chochos C. L., Choulis S. A.: How the structural deviations on the backbone of conjugated polymers influence their optoelectronic properties and photovoltaic performance. Progress in Polymer Science, 36, 13261414 (2011).

DOI: 10.1016/j.progpolymsci.2011.04.003

[17] Junkers T., Vandenbergh J., Adriaensens P., Lutsen L., Vanderzande D.: Synthesis of poly(p-phenylene vinylene) materials via the precursor routes. Polymer Chemistry, 3, 275-285 (2012). DOI: $10.1039 / \mathrm{C} 1 \mathrm{PY} 00345 \mathrm{C}$

[18] Cacialli F.: Conjugated and electroluminescent polymers. Current Opinion in Colloid and Interface Science, 4, 159-164 (1999). DOI: $10.1016 / \mathrm{S} 1359-0294(99) 00020-5$

[19] Jaballah N., Chemli M., Hriz K., Fave J-L., Jouini M., Majdoub M.: Blue-luminescent poly( $p$-phenylenevinylene) derivatives: Synthesis and effect of sidegroup size on the optical properties. European Polymer Journal, 47, 78-87 (2011).

DOI: $10.1016 /$ j.eurpolymj.2010.10.017

[20] Trad H., Majdoub M., Davenas J.: Soluble PPVs with few structural defects: Synthesis and characterization. Materials Science and Engineering: C, 26, 334-339 (2006).

DOI: 10.1016/j.msec.2005.10.041

[21] Pu L.: The study of chiral conjugated polymers. Acta Polymerica, 48, 116-141 (1997). DOI: $10.1002 /$ actp.1997.010480402

[22] Kotkar D., Joshi V., Ghosh P.: Towards chiral metals. Synthesis of chiral conducting polymers from optically active thiophene and pyrrole derivatives. Journal of the Chemical Society, Chemical Communications, 14, 917-918 (1988). DOI: $10.1039 / \mathrm{C} 39880000917$

[23] Lemaire M., Delabouglise D., Garreau R., Guy A., Roncali J.: Enantioselective chiral poly(thiophenes). Journal of the Chemical Society, Chemical Communications, 10, 658-661 (1988). DOI: $10.1039 / \mathrm{C} 39880000658$ 
[24] Moore J. S., Gorman C. B., Grubbs R. H.: Soluble, chiral polyacetylenes: Syntheses and investigation of their solution conformation. Journal of the American Chemical Society, 113, 1704-1712 (1991).

DOI: $\underline{10.1021 / \mathrm{ja} 00005 \mathrm{a} 039}$

[25] Feng L., Chen Z.: Synthesis and photoluminescent properties of polymer containing perylene and fluorene units. Polymer, 46, 3952-3956 (2005).

DOI: 10.1016/j.polymer.2005.02.102

[26] Melhuish W. H.: Quantum efficiencies of fluorescence of organic substances: Effect of solvent and concentration of the fluorescent solute. The Journal of Physical Chemistry, 65, 229-235 (1961).

DOI: $10.1021 / \mathrm{j} 100820 \mathrm{a} 009$

[27] Lauter U., Meyer W. H., Wegner G.: Molecular composites from rigid-rod poly( $p$-phenylene)s with oligo (oxyethylene) side chains as novel polymer electrolytes. Macromolecules, 30, 2092-2101 (1997). DOI: $10.1021 / \mathrm{ma} 961098 \mathrm{y}$

[28] Cope A. C., Shen T. Y.: The stereochemistry of 1,4: 3,6-dianhydrohexitol derivatives. Journal of the American Chemical Society, 78, 3177-3182 (1956). DOI: $10.1021 / \mathrm{ja} 01594 \mathrm{a} 055$

[29] Lemieux R. U., McInnes A. G.: The preferential tosylation of the endo-5-hydroxyl group of 1,4;3,6-dianhydRO-D-glucitol. Canadian Journal of Chemistry, 38, 136-140 (1960). DOI: $10.1139 / \mathrm{v} 60-015$

[30] Hriz K., Jaballah N., Chemli M., Fave J. L., Majdoub M.: Synthesis and characterization of new anthracenebased semiconducting polyethers. Journal of Applied Polymer Science, 119, 1443-1449 (2011). DOI: 10.1002/app.32659

[31] Hriz K., Chemli M., Jaballah N., Fave J-L., Majdoub M.: Synthesis, characterization and optical properties of distyrylanthracene-based polymers. High Performance Polymers, 23, 290-299 (2011).

DOI: $10.1177 / 0954008311405866$

[32] Gohil R. M.: Properties and strain hardening character of polyethylene terephthalate containing isosorbide. Polymer Engineering and Science, 49, 544-553 (2009). DOI: $10.1002 /$ pen. 20840

[33] Rouis A., Echabaane M., Sakly N., Dumazet-Bonnamour I., Ben Ouadaa H.: Electrochemical analysis of a PPV derivative thin film doped with $\beta$-ketoimine calix[4]arene in the dark and under illumination for the detection of $\mathrm{Hg}^{2+}$ ions. Synthetic Metals, 164, 78-87 (2013).

DOI: 10.1016/j.synthmet.2013.01.005

[34] van Oss C. J., Chaudhury M. K., Good R. J.: Monopolar surfaces. Advances in Colloid and Interface Science, 28, 35-64 (1987).

DOI: $10.1016 / 0001-8686(87) 80008-8$
[35] Ton-That C., Stockton G., Phillips M. R., Nguyen T-P., Huang C. H., Cojocaru A.: Luminescence properties of poly-(phenylene vinylene) derivatives. Polymer International, 57, 496-501 (2008).

DOI: $10.1002 /$ pi. 2373

[36] Noordover B. A. J., van Staalduinen V. G., Duchateau R., Koning C. E., van Benthem R. A. T. M., Mak M., Heise A., Frissen A. E., Haveren J. V.: Co- and terpolyesters based on isosorbide and succinic acid for coating applications: Synthesis and characterization. Biomacromolecules, 7, 3406-3416 (2006).

DOI: $10.1021 / \mathrm{bm} 060713 \mathrm{v}$

[37] Henari F. Z., Manaa H., Kretsch K. P., Blau W. J., Rost H., Pfeiffer S., Teuschel A., Tillmann H., Hörhold H. H.: Effective stimulated emission and excited state absorption measurements in the phenylene-vinylene oligomer (1,4-bis-(alpha-cyanostyryl)-2,5-dimethoxybenzene)). Chemical Physics Letters, 307, 163-166 (1999).

DOI: $10.1016 / \mathrm{S} 0009-2614(99) 00536-9$

[38] Peng K-Y., Chen S-A., Fann W-S., Chen S-H., Su AC.: Well-packed chains and aggregates in the emission mechanism of conjugated polymers. The Journal of Physical Chemistry B, 109, 9368-9373 (2005). DOI: 10.1021/jp044243f

[39] Huang Y. F., Shiu Y. J., Hsu J. H., Lin S. H., Su A. C., Peng K. Y., Chen S. A., Fann W. S.: Aggregate versus excimer emissions from poly(2,5-di- $n$-octyloxy-1,4phenylenevinylene). The Journal of Physical Chemistry C, 111, 5533-5540 (2007).

DOI: $10.1021 / \mathrm{jp} 067751 \mathrm{t}$

[40] Fan B., Sun Q., Song N., Wang H., Fan H., Li Y.: Electroluminescent properties of a partially-conjugated hyperbranched poly ( $p$-phenylene vinylene). Polymers for Advanced Technologies, 17, 145-149 (2006). DOI: 10.1002/pat.705

[41] Cheng M., Xiao Y., Yu W-L., Chen Z-K., Lai Y-H., Huang W.: Synthesis and characterization of a cyanosubstituted electroluminescent polymer with welldefined conjugation length. Thin Solid Films, 363, 110-113 (2000).

DOI: 10.1016/S0040-6090(99)01036-6

[42] Bredas J. L., Silbey R., Bordeaux D. S., Chance R. R.: Chain-length dependence of electronic and electrochemical properties of conjugated systems: Polyacetylene, polyphenylene, polythiophene, and polypyrrole. Journal of The American Chemical Society, 105, 6555-6559 (1983).

DOI: $10.1021 / \mathrm{ja} 00360 \mathrm{a} 004$ 